\title{
Atomic Layer Deposition of High-k Films on Graphene Surface
}

\author{
Hyeok Jae Lee, Kang Min Lee and Sang Woon Lee \\ Department of Energy Systems Research and Department of Physics, Ajou University \\ Suwon 16499, Republic of Korea \\ gurwoqod154@ajou.ac.kr; leekigg@ajou.ac.kr; slee01@ajou.ac.kr
}

\section{Extended Abstract}

Graphene has received considerable attentions because of its unique band structure and high electron mobility $(>20,000$ $\left.\mathrm{cm}^{2} / \mathrm{Vs}\right)$. Especially, the electronic characteristics of graphene is suitable as a channel material for the high performance metal-oxide-semiconductor field effect transistor. A growth of high-k film is necessary on graphene surface for top-gated graphene transistor.[1] However, graphene has no surface functional groups such as hydroxyl groups (-OH group) except for defects and graphene edges, which caused a failure of high-k film deposition on graphene surface even using state-of-the-art thin film deposition technique, i. e., atomic layer deposition (ALD). Thus, various methods were proposed to deposit dielectric films uniformly on graphene. For example, a growth of dielectric thin film by oxidation treatment after forming a metal seed layer using physical vapor deposition (PVD). In addition, a use of perylene-tetracarboxylic acid (PTCA), and pre$\mathrm{H}_{2} \mathrm{O}$ treatment were attempted. Unfortunately, impurities such as the perylene molecules remain on the graphene surface and the pre- $\mathrm{H}_{2} \mathrm{O}$ treatment and post-oxidation treatment become somewhat complicated.[2,3] In the meantime, enhancement of nucleation sites on graphene surface using ozone degraded the chemical state of graphene because of a defect formation on the graphene.[4] However, the chemical and electrical properties of the graphene must be preserved after the growth of highk films.[5]

Here, we propose a novel graphene surface treatment method that allows an enhanced nucleation and growth of high-k thin films by ALD via a graphene surface treatment using trimethylaluminum (TMA) and $\mathrm{H}_{2} \mathrm{O}$ prior to the deposition of $\mathrm{HfO}_{2}$ film on the graphene. Then, high-k thin films such as $\mathrm{Al}_{2} \mathrm{O}_{3}$ and $\mathrm{HfO}_{2}$ were deposited on the surface-treated graphene sample using ALD. These graphene surface treatment methods generate $\mathrm{Al}_{2} \mathrm{O}_{3}$ nuclei through physical adsorption on the surface of graphene, which enhanced the growth and nucleation of high-k film through ALD on the graphene surface. As a result, additional defects were not created after surface treatment on graphene and $\mathrm{HfO}_{2} \mathrm{ALD}$. And significant amount of Hf-C bond was observed after $\mathrm{HfO}_{2} \mathrm{ALD}$, which was decreased substantially with surface treatment. Nucleation delay in $\mathrm{HfO}_{2}$ ALD decreases from 70 to 10 ALD cycles with graphene surface treatment and dielectric constant of $\mathrm{HfO}_{2}$ film is higher using the surface treatment $(\mathrm{k} \sim 14.5)$ than that without the surface treatment $(\mathrm{k} \sim 5.6)$. Also, a leakage current (with a capacitor fabrication) was decreased by a factor of $10^{5}$ compared to without surface treatment. The graphene surface treatment method enhanced the nucleation and the electrical properties of the $\mathrm{HfO}_{2}$ film without deteriorating the properties of graphene, thus providing promising opportunities in graphene electronics.

\section{References}

[1] F. Schwierz, "Graphene transistors," Nature nanotechnology, vol. 5, pp. 487-496, 2010.

[2] B. Fallahazad, K. Lee, G. Lian, S. Kim, C. Corbet, D. Ferrer, L. Colombo, and E. Tutuc, "Scaling of $\mathrm{Al}_{2} \mathrm{O}_{3}$ dielectric for graphene field-effect transistors," Applied Physics Letters, vol. 100, p. 093112, 2012.

[3] X. Wang, S. Tabakman, and H. Dai, "Atomic layer deposition of metal oxides on pristine and functionalized graphene," Journal of the American Chemical Society, vol. 130, pp. 8152-8153, 2008

[4] B. Lee, S.-Y. Park, H.-C. Kim, K. Cho, E. M. Vogel, M. J. Kim, R. M. Wallace, and J. Kim, "Conformal $\mathrm{Al}_{2} \mathrm{O}_{3}$ dielectric layer deposited by atomic layer deposition for graphene-based nanoelectronics," Applied Physics Letters, vol. 92, p. 203102, 2008.

[5] Y. H. Park, M. H. Kim, S. B. Kim, H. J. Jung, K. Chae, Y. H. Ahn, J.-Y. Park, F. Rotermund, S. W. Lee, "Enhanced Nucleation of High-K Dielectrics on Graphene by Atomic Layer Deposition," Chemistry of Materials, vol. 28, pp. 7268$7275,2016$. 